\title{
Bed bugs: The move to humans as hosts
}

\author{
Benoit Talbot $^{\mathrm{a} \star}$, Nusha Keyghobadi ${ }^{\mathrm{b}}$, and Brock Fenton ${ }^{\mathrm{b}}$ \\ ${ }^{a}$ School of Epidemiology and Public Health, University of Ottawa, Ottawa, ON K1G 5Z3, Canada; \\ ${ }^{\mathrm{b}}$ Department of Biology, University of Western Ontario, London, ON N6A 5B7, Canada \\ *benoit.talbot@uottawa.ca
}

\begin{abstract}
Cimicid insects, bed bugs and their allies, include about 100 species of blood-feeding ectoparasites. Among them, a few have become widespread and abundant pests of humans. Cimicids vary in their degree of specialization to hosts. Whereas most species specialize on insectivorous birds or bats, the common bed bug can feed on a range of distantly related host species, such as bats, humans, and chickens. We suggest that association with humans and generalism in bed bugs led to fundamentally different living conditions that fostered rapid growth and expansion of their populations. We propose that the evolutionary and ecological success of common bed bugs reflected exploitation of large homeothermic hosts (humans) that sheltered in buildings. This was a departure from congeners whose hosts are much smaller and often heterothermic. We argue that interesting insights into the biology of pest species may be obtained using an integrated view of their ecology and evolution.
\end{abstract}

Key words: bed bug, Cimicidae, evolutionary biology, parasite-host interactions, public health

The common bed bug (Cimex lectularius Linnaeus, 1758) is a widespread and locally abundant pest of humans. It is one of about 100 species of cimicid insects, most of which are small, flightless, obligate blood feeders (Usinger 1966; Reinhardt and Siva-Jothy 2007). Males and females eat only blood and a blood meal is required for egg production, mating, growth, and development to adulthood. Most cimicids consume the blood of bats (Molossidae and Vespertilionidae) or birds (Apodidae-swifts; Hirundinidae-swallows). Some populations of common bed bugs, however, have adapted to living with and on humans, and perhaps chickens (Reinhardt and Siva-Jothy 2007). About once a week, captive adult cimicids feed for 10-20 min. When not feeding, cimicids hide in cracks and crevices (Usinger 1966; Reinhardt and Siva-Jothy 2007). Although they spend most of their time off of their hosts' bodies, cimicids are often considered ectoparasites because they remain within the host's roost, nest, or dwelling. According to other definitions, however, they could be classified as micropredatory blood-suckers (Balashov 2006).

Cimicids exhibit variation in the range of host taxa used by any species (e.g., Usinger 1966; Reinhardt and Siva-Jothy 2007). Two subfamilies (Primicimicinae and Latrocimicinae) use New World bats as hosts, whereas two others (Cacodminae and Afrocimicinae) use Old World bats. The hosts of Haematosiphoninae are a variety of birds in the New World. Cimex lectularius and Cimex hemipterus occur mainly on humans, but can survive on chickens (Benoit 2011; Criado et al. 2011). Furthermore, one lineage of C. lectularius occurs on several species of Old World bats.

Among parasites, weak generalists use several closely related species of hosts (Mazé-Guilmo et al. 2016). These may be more efficient feeders and have higher fecundity when feeding on preferred hosts (Reinhardt and Siva-Jothy 2007). Strong generalists are species using several phylogenetically distinct

hosts (Mazé-Guilmo et al. 2016). Only a few cimicids, such as the common bed bug and possibly the 
tropical bed bug C. hemipterus, appear to be strong generalists. Most other cimicids are weak generalists. Molecular data provide evidence for adaptation by cimicids to different hosts. There has been a significant divergence among and within cimicid species at genes coding for salivary proteins, which are important for blood feeding. Divergence has been recorded among different cimicid species associating with different hosts across the world (Talbot et al. 2017). The same is true among populations of a single species associating with different bat species in North America (Talbot et al. 2018). Flexibility in choice of hosts may benefit strong generalists when conditions change. The purpose of this paper is to speculate about key ecological changes that some cimicids would have encountered as they began, and then continued, to include humans as preferred hosts. This meant a transition from weak to strong generalism, which resulted in population growth and emergence of a worldwide human pest.

The first record of a cimicid-like insect comes from Cretaceous amber from Myanmar (Grimaldi et al. 2002). Bats appeared somewhat later (Fenton and Simmons 2014). Like others, we propose that bats were early hosts for cimicids (Usinger 1966; Balvín et al. 2012; Booth et al. 2015). The importance of cimicids to the life cycles of trypanosomes associated with bats (Bower and Woo 1981) suggests a long association between bats and these insects. A stable transition of some cimicids to human hosts possibly occurred in the Pleistocene epoch. In Eurasia, although the common bed bug occurs on a range of insectivorous bat species, populations of one lineage associate mostly with humans (Balvín et al. 2012, 2015; Booth et al. 2015). This lineage appears to have diverged from the rest of the group about 245000 years ago. By the late Pleistocene and early Holocene, the association between cimicids and humans appears to have become widespread. Reinhardt and Siva-Jothy (2007) reported evidence of at least 4000 years of association between humans and cimicids. Recent discoveries in western North America suggest that at least three cimicid species and humans were in contact in shelter caves since about 11000 years ago (Adams and Jenkins 2017).

Despite their small individual body size, communal roosting makes groups of bats a relatively large and stable food supply for cimicids. In summer, vespertilionid and molossid hosts typically roost in dark crevices and hollows (e.g., Willis and Brigham 2007). Depending upon the species, these bats weigh about 5-30 g and often roost in groups. In a roost, bats themselves may be as important to other bats as the exact nature of the roost. This reflects the clear thermoregulatory benefit to clustering with others (Brown 1999; Willis et al. 2005; Pretzlaff et al. 2010). Clusters of day-roosting bats in summer are usually conspecifics, and regular roost-switching is taken to reflect a fission-fusion social organization (Willis and Brigham 2004). Nursery colonies consist of pregnant and lactating females with their dependent young. These groups represent a ready supply of blood for cimicids. Movements of individuals and fission-fusion social organization means that cimicids living in any bat roost are mainly exposed to one species, but may feed on several individuals over the summer. Many species of bats show different levels of roost fidelity, perhaps making these mammals predictable food sources (Lewis 1995). However, high roost-switching frequency in many species is hypothesized to be a strategy to reduce parasitism (Willis and Brigham 2004; Bartonička and Rủžičková 2013). Therefore, host fidelity in most cimicid species is probably more a function of bat behavior than choices made by cimicids.

Although offset to some extent by communal roosting behavior, the size of individual bats and aspects of their thermoregulation may, nonetheless, be limiting factors for blood-feeding ectoparasites. The body mass of unfed adult female common beg bugs is $5 \mathrm{mg}$, on average, but they eat, on average, $7.6 \mathrm{mg}$ in any blood meal (Usinger 1966). In mammals, blood constitutes about $10 \%$ of body mass, so a $10 \mathrm{~g}$ adult bat holds approximately 50000 times less blood than a human. Humans are also more consistently homeothermic than vespertilionid or molossid bats. Although a bat's body temperature is elevated when flying and foraging, its metabolic rate, and consequently body temperature, are reduced 
after returning to its roost (Stawski et al. 2014). For individuals that enter torpor, declines in body temperature can be rapid, within 30 min of returning to the roost (Audet and Fenton 1988). Based on efforts to collect blood from bats, biologists have learned that it is difficult to obtain samples from torpid individuals (Martin and Stehn 1977). If body temperature affects blood flow, and possibly, as a consequence, cimicid blood feeding behavior (DeVries et al. 2016), cimicids feeding on bats likely have relatively little time to efficiently consume blood. The behavior of cimicids and their bat hosts suggests that effective feeding must occur as soon as possible after bats return to the roost. Thus, heterothermy may reduce the resource value of bats as hosts for cimicids compared with consistently homeothermic vertebrates such as birds or humans. Overall, the combination of small size and heterothermy could mean that for cimicids, bats represent a more limiting resource.

By occupying cracks, crevices and hollows in human dwellings, bats would have brought their cimicid parasites into close contact with humans (Usinger 1966). The proximity of roosting bats and their cimicids to people likely created opportunities for cimicids to adapt to new hosts. There are contemporary records of cimicid individuals from species commonly associated with bats, such as C. adjunctus, C. pipistrelli, and C. pilosellus, occasionally feeding on humans visiting or residing near a bat roost (Goddard et al. 2012). Unlike many insects, female cimicids cannot store seminal fluid but instead may use it for nutrition (Hinton 1964). For this reason, female cimicids may be able to survive in the absence of the host for a limited time. Nonetheless, in the event of bats not returning to a roost for an extended duration (Bartonička and Rủžičková 2013), humans would constitute a convenient alternative food source. Mechanisms underlying reproductive isolation, which could subsequently lead to speciation, have been explored in bat- and human-associated C. lectularius lineages in Europe (Wawrocka et al. 2015; DeVries et al. 2017).

For a blood-feeding ectoparasite, transition to a relatively large, consistently homeothermic host could dramatically increase resource availability and carrying capacity (Morand et al. 2006). Phylogeographic data for European C. lectularius suggests 20\% higher effective population sizes in lineages associated with humans compared with the ancestral lineage that is mainly associated with bats (Balvín et al. 2012). In contrast, C. adjunctus, a North American species typically associated exclusively with bats, shows an overall decrease of effective population size across the continent over the last 300000 years (Talbot et al. 2016). Overall, phylogeographic analyses suggest higher effective population size and potentially greater population stability over the long term for cimicid lineages associated with humans compared with bats. Thus, humans are large, dependable sources of blood, and constitute a stable food source for cimicids. Furthermore, human dwellings offer many crevice retreats for cimicids and a stable environment.

In conclusion, we argue that key differences between bat and human hosts, particularly in body size and thermoregulation, allowed the ecological release of cimicid populations that moved to humans as hosts. These differences contributed in large part to the evolutionary and ecological success of at least one lineage of Cimicidae that has emerged as the cosmopolitan common bed bug. We suggest that this example illustrates the interesting insights into the biology of pest species that may be obtained using an integrated view of their ecology and evolution.

\section{Acknowledgements}

We thank Western University, Natural Sciences and Engineering Research Council of Canada, Ontario Student Assistance Program, and Schmeelk Canada Foundation for providing financial support to Benoit Talbot during his doctoral degree, during which prior research leading to this editorial was produced. We thank R. Mark Brigham for his useful suggestions on one of the versions of the manuscript. 


\section{Author contributions}

BT, NK, and BF conceived and designed the study. BT, NK, and BF drafted or revised the manuscript.

\section{Competing interests}

$\mathrm{BF}$ is currently serving as a Subject Editor for FACETS, but was not involved in review or editorial decisions regarding this manuscript.

\section{Data availability statement}

All relevant data are within the paper.

\section{References}

Adams ME, and Jenkins DL. 2017. An early Holocene record of Cimex (Hemiptera: Cimicidae) from western North America. Journal of Medical Entomology, 54(4): 934-944. PMID: 28399298 DOI: $10.1093 / \mathrm{jme} / \mathrm{tjx} 057$

Audet D, and Fenton MB. 1988. Heterothermy and the use of torpor by the bat Eptesicus fuscus (Chiroptera: Vespertilionidae): a field study. Physiological and Biochemical Zoology, 61(3): 197-204. DOI: 10.1086/physzool.61.3.30161232

Balashov YS. 2006. Types of parasitism of acarines and insects on terrestrial vertebrates. Entomological Review, 86(8): 957-971. DOI: 10.1134/S0013873806080112

Balvín O, Munclinger P, Kratochvíl L, and Vilímová J. 2012. Mitochondrial DNA and morphology show independent evolutionary histories of bedbug Cimex lectularius (Heteroptera: Cimicidae) on bats and humans. Parasitology Research, 111(1): 457-469. PMID: 22392133 DOI: 10.1007/ s00436-012-2862-5

Balvín O, Roth S, and Vilímová J. 2015. Molecular evidence places the swallow bug genus Oeciacus Stål within the bat and bed bug genus Cimex Linnaeus (Heteroptera: Cimicidae). Systematic Entomology, 40: 652-665. DOI: 10.1111/syen.12127

Bartonička T, and Růžičková L. 2013. Recolonization of bat roost by bat bugs (Cimex pipistrelli): could parasite load be a cause of bat roost switching? Parasitology Research, 112(4): 1615-1622. PMID: 23385971 DOI: 10.1007/s00436-013-3316-4

Benoit J. 2011. Stress tolerance of bed bugs: a review of factors that cause trauma to Cimex lectularius and C. hemipterus. Insects, 2(4): 151-172. PMID: 26467619 DOI: 10.3390/insects2020151

Booth W, Balvín O, Vargo EL, Vilímová J, and Schal C. 2015. Host association drives genetic divergence in the bed bug, Cimex lectularius. Molecular Ecology, 24(5): 980-992. PMID: 25611460 DOI: $10.1111 / \mathrm{mec} .13086$

Bower SM, and Woo PTK. 1981. Development of Trypanosoma (Schizotrypanum) hedricki in Cimex brevis (Hemiptera: Cimicidae). Canadian Journal of Zoology, 59(3): 546-554. DOI: 10.1139/ z81-078

Brown CR. 1999. Metabolism and thermoregulation of individual and clustered long-fingered bats, Miniopterus schreibersii, and the implications for roosting. South African Journal of Zoology, 34(4): 166-172. DOI: 10.1080/02541858.1999.11448505 
Criado PR, Belda W Jr, Criado RFJ, Vasconcelos e Silva R, and Vasconcellos C. 2011. Bedbugs (Cimicidae infestation): the worldwide renaissance of an old partner of human kind. The Brazilian Journal of Infectious Diseases, 15(1): 74-80. PMID: 21412594 DOI: 10.1016/S1413-8670(11)70144-1

DeVries ZC, Mick R, and Schal C. 2016. Feel the heat: activation, orientation and feeding responses of bed bugs to targets at different temperatures. Journal of Experimental Biology, 219(23): 3773-3780. PMID: 27688050 DOI: 10.1242/jeb.143487

DeVries ZC, Mick R, Balvín O, and Schal C. 2017. Aggregation behavior and reproductive compatibility in the family Cimicidae. Scientific Reports, 7(1): 13163. PMID: 29030574 DOI: $10.1038 /$ s41598-017-12735-3

Fenton MB, and Simmons NB. 2014. Bats: a world of science and mystery. The University of Chicago Press, Chicago, Illinois.

Goddard J, Baker GT, Ferrari FG, and Ferrari C. 2012. Bed bugs (Cimex lectularius) and bat bugs (several Cimex species): a confusing issue. Outlooks on Pest Management, 23(3): 125-127. DOI: $10.1564 / 23$ jun09

Grimaldi DA, Engel MS, and Nascimbene PC. 2002. Fossiliferous Cretaceous amber from Myanmar (Burma): its rediscovery, biotic diversity, and paleontological significance. American Museum Novitates, 1956(3361): 1-72. DOI: 10.1206/0003-0082(2002)361<0001:FCAFMB > 2.0.CO;2

Hinton HE. 1964. Sperm transfer in insects and the evolution of haemocoelic insemination. In Proceedings of the Second Symposium of the Royal Entomological Society of London, Imperial College, London, UK, 19-20 September 1963. pp. 95-107.

Lewis SE. 1995. Roost fidelity of bats: a review. Journal of Mammalogy, 76(2): 481-496. DOI: $10.2307 /$ 1382357

Martin KH, and Stehn RA. 1977. Blood and spleen response to isolation and cold stress in little brown bats, Myotis lucifugus. Acta Zoologica, 58(1): 53-59. DOI: 10.1111/j.1463-6395.1977.tb00236.x

Mazé-Guilmo E, Blanchet S, McCoy KD, and Loot G. 2016. Host dispersal as the driver of parasite genetic structure: a paradigm lost? Ecology Letters, 19(3): 336-347. PMID: 26843399 DOI: 10.1111/ ele.12564

Morand S, Krasnov BR, and Poulin R (Editors). 2006. Micromammals and macroparasites: from evolutionary ecology to management. Springer Publishing, New York, New York.

Pretzlaff I, Kerth G, and Dausmann KH. 2010. Communally breeding bats use physiological and behavioural adjustments to optimise daily energy expenditure. Naturwissenschaften, 97(4): 353-363. PMID: 20143039 DOI: 10.1007/s00114-010-0647-1

Reinhardt K, and Siva-Jothy MT. 2007. Biology of the bed bugs (Cimicidae). Annual Review of Entomology, 52(1): 351-374. PMID: 16968204 DOI: 10.1146/annurev.ento.52.040306.133913

Stawski C, Willis CKR, and Geiser F. 2014. The importance of temporal heterothermy in bats. Journal of Zoology, 292(2): 86-100. DOI: 10.1111/jzo.12105

Talbot B, Vonhof MJ, Broders HG, Fenton B, and Keyghobadi N. 2016. Range-wide genetic structure and demographic history in the bat ectoparasite Cimex adjunctus. BMC Evolutionary Biology, 16(1): 268. PMID: 27927166 DOI: 10.1186/s12862-016-0839-1 
Talbot B, Balvín O, Vonhof MJ, Broders HG, Fenton B, and Keyghobadi N. 2017. Host association and selection on salivary protein genes in bed bugs and related blood-feeding ectoparasites. Royal Society Open Science, 4(6): 170446. PMID: 28680688 DOI: 10.1098/rsos.170446

Talbot B, Vonhof MJ, Broders HG, Fenton B, and Keyghobadi N. 2018. Host association influences variation at salivary protein genes in the bat ectoparasite Cimex adjunctus. Journal of Evolutionary Biology, 31(5): 753-763. PMID: 29543391 DOI: 10.1111/jeb.13265

Usinger RL. 1966. Monograph of Cimicidae (Hemiptera, Heteroptera). Entomological Society of America, Annapolis, Maryland.

Wawrocka K, Balvín O, and Bartonička T. 2015. Reproduction barrier between two lineages of bed bug (Cimex lectularius) (Heteroptera: Cimicidae). Parasitology Research, 114(8): 3019-3025. PMID: 25952703 DOI: 10.1007/s00436-015-4504-1

Willis CKR, and Brigham RM. 2004. Roost switching, roost sharing and social cohesion: forestdwelling big brown bats, Eptesicus fuscus, conform to the fission-fusion model. Animal Behaviour, 68(3): 495-505. DOI: 10.1016/j.anbehav.2003.08.028

Willis CKR, and Brigham RM. 2007. Social thermoregulation exerts more influence than microclimate on forest roost preferences by a cavity-dwelling bat. Behavioral Ecology and Sociobiology, 62(1): 97-108. DOI: $10.1007 /$ s00265-007-0442-y

Willis CKR, Lane JE, Liknes ET, Swanson DL, and Brigham RM. 2005. Thermal energetics of female big brown bats (Eptesicus fuscus). Canadian Journal of Zoology, 83(6): 871-879. DOI: 10.1139/ z05-074 